\title{
EuCLIS - a web based European climate information system
}

\author{
P. Bissolli \\ German Meteorological Service, Offenbach, Germany
}

Received: 15 February 2007 - Revised: 5 June 2007 - Accepted: 6 August 2007 - Published: 19 September 2007

\begin{abstract}
The German Meteorological Service (Deutscher Wetterdienst) is presently developing a European climate information system (EuCLIS) which is presented here. The aim of this system is to distribute climate monitoring information of the area of the WMO (World Meteorological Organisation) Region VI (Europe and the Middle East) via the web. EuCLIS will be the successor of the already existing platform GCMP (Generate Climate Monitoring Products) which emerged from a project of the ECSN (European Climate Support Network). Climate monitoring information can be all kind of maps, diagrams and texts describing the variability of climate variables in space and time. It can be provided by the public national meteorological and hydrological services of all countries of the WMO Region VI. The main advantage is to have one common efficient climate monitoring distribution system for the whole Region, but the individual meteorological and hydrological services have the possibility to administrate their products on their own. EuCLIS considers the WMO metadata standard and modern web portal technology. In an advanced state, EuCLIS is intended to be used as a platform for a function of a Regional Climate Centre for Climate Monitoring which is currently planned at the WMO.
\end{abstract}

\section{Introduction}

Climate change does not stop at national borders; this has become clear since the global warming debate. However, we also know that the climate does not change in the same way across the earth. Even within Europe, which has a very heterogeneous climate, a large variability of climate change in space and time occurred in the past decades as can be seen on the basis of observations, and this is also to be expected in future based on climate change scenarios as analysed e.g. by Scherrer et al. (2005) for Central Europe. It is thus essential to establish an extensive continuous operational climate monitoring, not only on a global or a national scale, but also for a region covering a continent like Europe and adjacent areas.

In Europe, mainly the governmental National Meteorological and Hydrological Services (NMHSs) are in charge of providing regularly operational climate monitoring information to the public. During the recent years, when the internet became more and more the primary source for distributing up-to-date information, many NMHSs built up web-based climate information systems. One example among many oth- ers is the climate information system KLIS at the German Meteorological Service, described by Behrendt and Rösner (1997), which is now redesigned due to a change to a new modern web portal technology. Beyond Europe, other modern climate information systems are described, e.g. for the USA (Hubbard et al., 2004) or China (Shang et al., 2001).

Since the provision of climate monitoring information is under national responsibility in Europe, the national climate monitoring systems are more or less well established. However, for Europe as a whole, this is not on the same level. Although a lot of climate data are distributed via the Global Telecommunication System (GTS) or via the web, and further climate data from satellites are available for the public, each NMHS has only limited information about the data for whole Europe. Many NMHSs have their own national climate data networks, and their data are not distributed worldwide because of technical or political reasons. In addition, in many cases also the resources in the NMHSs are not available to produce extensive climate monitoring for whole Europe to the same extent as on the national level.

Correspondence to: P. Bissolli

(peter.bissolli@dwd.de)

Published by Copernicus Publications. 


\section{The GCMP project of the European Climate Sup- port Network}

The European Climate Support Network (ECSN) is a branch of EUMETNET (Network of European Meteorological Services). After recognising an increase of requirements concerning the task of climate monitoring and the need for an improvement of the co-ordination of the various national activities, the project "Generate Climate Monitoring Products" (GCMP) was proposed to ECSN in the year 1998 and approved one year after. The objective of the project was to set up an internet platform to provide climate monitoring information, including extreme weather events, in a quasi operational way, covering the area of Europe. The basis for this information had to be given by products generated by the NMHSs. The German Meteorological Service was put in charge with the co-ordination of the project.

Up to now (2007), the internet platform has been developed and is still running in a quasi-operational state. The products consist of descriptions of significant weather events (texts) and maps and diagrams of daily and monthly mean temperature, precipitation, sunshine, and various other climate elements, provided regularly. The main technical feature of the GCMP platform is the following principle: All the climate monitoring products are located on the web servers of the NMHSs. Only the GCMP homepage is located on the web server of the host (German Meteorological Service). Any product request is just realised by linking from the GCMP homepage to the product web page of the concerned NMHS. Thus, a duplication of product generation can be avoided, the products can remain on the national web servers, and the user has easy access to all the products starting from one common homepage. Presently 19 ECSN countries contribute regularly products to GCMP. The products are accessible under http://www.gcmp.dwd.de. An example is shown in Fig. 1.

One main shortcoming of GCMP is the fact, that no complete automation has been realised. All new URLs have to be attributed manually by the host. This takes time resources for checking, changing and updating the products regularly, and new products are only available with a certain delay.

\section{The successor system EuCLIS}

\subsection{Requirements to the new system}

To improve the present operational GCMP system and to take advantage of modern web technology, it was decided to establish a new web platform as successor system of GCMP, called EuCLIS (European Climate Information System). The requirements to the new system were the following:

- The basic idea of GCMP should remain: access to all available climate monitoring products of the participating countries from one homepage; no product duplication, only links to existing products.
- The new system should offer at least the same products as GCMP, but it should be open for any new products.

- It is desirable to extend the number of participating countries (presently 19 in GCMP). The target area is the so called WMO Region VI which comprises the whole European continent, excluding the Ural in the east, but including some countries in the Middle East, and towards the west, including Greenland and parts of the North Atlantic (Fig. 2).

- Potentially, this would require the participation of NMHSs from 50 countries (including Montenegro which has its own NMHS now) which is of course up to the decision of the individual NMHSs.

- It is intended that the NMHSs will have the opportunity to administrate their products themselves in the new system, in contrast to the present GCMP. The advantage is, that the individual NMHSs could decide anytime at once, which new products they want to insert or which of the existing ones should be changed or deleted. After insertion of the product metadata and URLs, the product distribution should be done automatically without any further manual actions. This will speed up the provision of products. The administration should of course be easy to handle, so that changes can be carried out easily and quickly.

- The navigation is to be improved compared to GCMP. Some additional functions are also to be realised, e.g. search, sort and group functions for the products.

- The new system should offer an open and a userrestricted area. That way, it is possible to distribute products which are not public to a restricted user community while there is also a public domain for products which are always freely accessible for everyone.

- The possibility of installing versions in different languages should be possible. The first version will be in English like GCMP, but the system should be extendable for other language versions as well if requested.

- The metadata information has to be stored in XML files which should accomplish the ISO standards 19115 and 19139 (see e.g. ISO catalogue, 2007) used by the WMO.

- The new system has to be integrated into the new web portal of the German Meteorological service (a BEA weblogic portal with portlet technology, see http://de. bea.com/products/weblogic/) and should accomplish all modern general requirements on web sites (e.g. access for handicapped persons). 


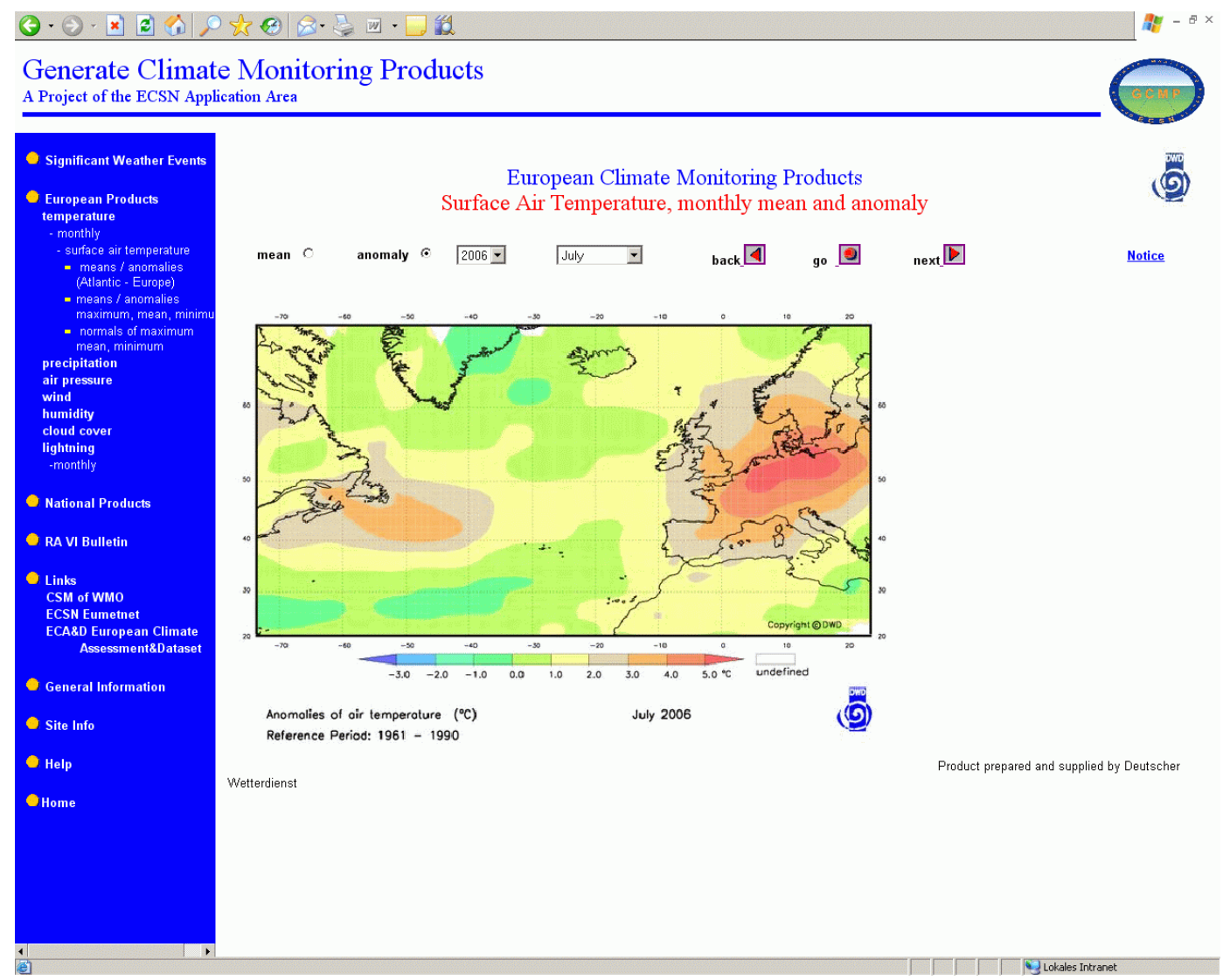

Figure 1. Browser snapshot of the GCMP system displaying the monthly mean near-surface air temperature anomalies for July 2006 relative to the 1961-1990 base period. (Source: German Meteorological Service, based on CLIMAT messages and ship data). This month was characterised by extreme high temperature anomalies especially over Central Europe; in many areas this was the warmest ever July since the beginning of measurements.

\subsection{Technical realisation}

A technical concept was set up to describe a solution which meets all requirements listed above. First a standard dynamical web application was developed which was adapted for integration into the new web portal.

The new web portal is based on a special technology which offers many advantages compared to previous web technologies. One main advantage of portals is the possibility to combine various processes and pieces of functionality into one single web interface. Before portals, web users could visit only one page at a time. With portals, users are able to view various sites at the same time. The technology behind this system makes use of a fragmentation of web sites. Such fragments are displayed in so called portlets (a kind of "windows"). These portlets can be combined or separated from each other as needed for various purposes, and they also can communicate to each other via an application in the background. A second advantage of portals is the personalisation of web sites realised by a single sign-on. Users log in for the whole portal and get to see their customised portlets dependent on their individual user profiles.
Within a portlet, a navigation can lead either to a dynamical application (e.g. creating or displaying graphics chosen by a drop-down menu) or to static web pages (displaying just fixed texts or graphics). Static web pages were generated using the Government Site Builder (a content manager system solution developed for governmental agencies in Germany (Bundesverwaltungsamt, 2007). The Government Site Builder is a very flexible and user-friendly tool for generating web pages using simple forms.

The web application itself is written in Java programming language and meets the J2EE standard (http://java.sun.com/ j2ee/1.4). One main feature of the software architecture is the separation between two layers: the so called business logic for transferring the URLs and all the metadata needed and the presentation logic for displaying the products. The software architecture makes use of various java tools; details about these tools require a deeper knowledge of java and are beyond the scope of this paper.

All interfaces are provided by the BEA Weblogic Server via the browser per http. Two portlets are created for EuCLIS: one for the users and one for the administrators. 


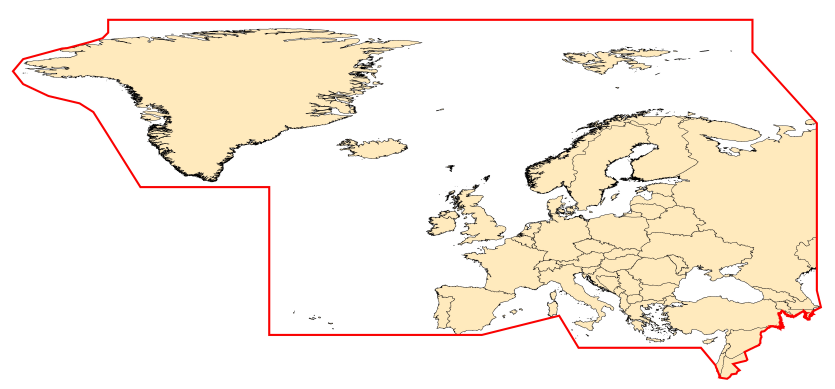

Figure 2. The WMO RA VI Region.

The user portlet is just for displaying all the products; whereas in the administrator portlet, new products can be inserted and existing ones can be changed or deleted ("Admin tool"). There are four different user groups: open and closed users, local administrators (at least one for each participating NMHS) and the main administrator (a person of the host's staff). The user portlet can be seen by everybody, but the open user can not see those products in the user portlet which are restricted to a limited user community, whereas the closed user can see all products. The administrator portlet can only be seen by the local administrators and the main administrator, and the local administrators have only access to products of their own country. Thus, only the administrators will be able to perform any changes of the products. The authentication of the user is realised by logging in with password.

All the configuration and metadata are stored on XML files which have to accomplish the WMO ISO standards 19115 and 19139. ISO 19115 defines the meta information itself. It consists of more than 400 meta data elements about the content of the data, its spatial and time references, data quality and access. In practice not all elements are defined, but there is a mandatory minimum of meta information consisting of about 20 elements. ISO 19139 defines the structure of the XML metadata files. In EuCLIS, four groups of meta information are defined:

- general product information: title and description of the content of data, keywords;

- specific product information: climate element or subelement, time interval and average, data base, reference period, boundary coordinates;

- file information: date, format, version, size;

- contact information: name, institution and coordinates of the contact person of the products.

The XML files are stored on a file server at the host, a NAS. The configuration files determine the navigation of the home page and the product configuration given by the meta information. Whenever the administrator adds or changes any product specification (meta information), this is not to be done on the XML file directly; all the changes can be entered into a form, and the system stores all the information automatically in XML files. Thus, the administrators need not to be familiar with XML codes.

The products themselves (text and graphic files) are located on the web servers of the participating NMHSs like before in GCMP.

\subsection{Product categories}

Mainly the same product categories as in GCMP are used also for EuCLIS, only the structure has been a little bit simplified. The main categories are the following:

- Significant weather events: texts of climatological assessment of remarkable weather and climate events, e.g. heavy storms, heavy precipitation with flooding, heat waves, longer cold spells etc. It is to be decided by the NMHSs which events are chosen.

- Products in general: These are all kind of maps and diagrams describing climate monitoring, typically daily, monthly, seasonal or annual means of various climate elements (at least air temperature and precipitation) and their anomalies from a long-term mean (normal) of a reference period (mostly 1961-1990 as recommended by the WMO). National products cover only one country while so called "European" products cover more than one country, whole Europe or even the whole RA VI Region, although the data base (station density) might be smaller than on the national level.

- Links: Some special products from other providers which do not fit into the other categories, e.g. special publications, other climate monitoring sites, data providers etc.

In EuCLIS, it is not intended to provide direct access to climate data (station or grid values), only to post processed products (maps, text evaluations), since data access is provided elsewhere (e.g. by ECA\&D: http://eca.knmi.nl, UNIDART: http://www.dwd.de/UNIDART).

\section{Conclusions and outlook}

EuCLIS will be developed as a joint platform for distributing climate monitoring information via the web for a large heterogeneous region (WMO RA VI Region). The modern web technology enables all the possibilities for a fast and easy transfer of all various kinds of climate monitoring products. However, the success of such an activity is strongly dependent on the continuous participation of a sufficient number of NMHSs. Up to now, this concept has worked very well for GCMP since some years and gives hope that this can be continued in future as well.

Further product development activities are also ongoing in the meantime. The number of products available on national 
climate monitoring web sites is increasing and also the methods of climate map productions are improving, taking e.g. geographical information systems (GIS) into account. There is already a widespread use of GIS applications in climatology as discussed e.g. within the COST Action 719 (Dobesch et al., 2007).

Presently, the integration of the EuCLIS web application into the new web portal is almost complete. First internal tests have been carried out and some bugs have been detected and solved. In a next step, another test phase (beta testing) will take place soon. During this phase, voluntary local administrators of some NMHSs will insert some products into the system and test its response and performance under operational conditions. It is planned that EuCLIS will become fully operational in 2008 and replace GCMP then. However, it is assured that GCMP will not switched off before EuCLIS is able to provide at least the same range of products as GCMP.

For the future, it is envisaged to use EuCLIS as a platform for a so called Regional Climate Centre (RCC, see Bissolli et al. (2005) for a rough overview) for Climate Monitoring of the WMO to exchange all kind of climate monitoring information between the NMHSs of the WMO Region VI which can be further distributed to the public.

Acknowledgements. The author thanks the guest editors M. Dolinar and I. Dyras for initiating these proceedings and organising the reviewing process, and also the reviewers for constructive remarks on the paper. And last, but not least thanks a lot to all participating NMHSs of the GCMP platform who contributed regularly their climate monitoring products over some years now. This was a strong encouragement to initiate the EuCLIS project as well and to continue the good cooperation which we had up to now.

Edited by: M. Dolinar and I. Dyras

Reviewed by: F. J. M. van der Wel and another anonymous referee

\section{References}

Behrendt, J. and Rösner, S.: The climate information system KLIS at the DWD, Climate and Environmental Database Systems, Workshop, Hamburg, Germany, edited by: Lautenschlager, M., Boston, 1997.

Bissolli, P., Hechler, P., and Nitsche H.: WMO Regional Climate Centres - concept and DWD's potential role, Deutscher Wetterdienst, Climate Status Report 2005.

Bundesverwaltungsamt of the German government: Government Site Builder (in German), Cologne, http://www. government-site-builder.de, 2007.

Dobesch, H., Dumolard, P., and Dyras, I. (Eds.): Spatial interpolation for climate data, ISTE Ltd., London, 2007.

Hubbard, K. G., DeGaetano, A. T., and Robbins, K. D.: A modern applied climate information system, B. Am. Meteor. Soc., 85, 811-812, 2004.

ISO Catalogue 2007: available at the Organization for Standardization (ISO), ISBN 978-92-67-10433-1, 2007.

Scherrer, S. C., Appenzeller, C., Liniger, M. A., and Schär, C.: European temperature distribution changes in observations and climate change scenarios, Geophys. Res. Lett., 32, L19705, doi:10.1029/2005GL024108, 2005.

Shang, Z., Gao, Q., and Yang, D.: The climate information system of China, Clim. Res., 18, 229-247, 2001. 